\title{
Role of Ultrasound for the Diagnosis of Uterine Prolapse in Women: A Systemic Review
}

\author{
Sadiq Amin Qaisrani* \\ The University of Lahore, 1-KM Defence Road, Lahore, Pakistan \\ Syeda Khadija-Tul-Sughra \\ The University of Lahore, 1-Km Defense Road Lahore ,Pakistan \\ Muhammad Rohail Amjad \\ The University of Lahore, 1-Km Defense Road Lahore ,Pakistan \\ Hira Riffat \\ The University of Lahore, 1-Km Defense Road Lahore ,Pakistan
}

\begin{abstract}
Background: Uterine prolapse is the herniation of the uterus into or beyond the vagina as a result of failure of the ligamentous and fascia supports. It often coexists with prolapse of the vaginal walls, involving the bladder or rectum.

Objective: The aim of this study is to evaluate the role of ultrasound for the diagnosis of uterine prolapse in young women.

Methodology: In this systematic review data extracted by Medline, PubMed, science direct and Obs-gyne online library that were queried for studies published between 2000 to 2021 by using specific MeSH terms. In this systematic article we reviewed 10 retrospective style analytical studies for the collection of data.

Results: 10 studies conducted between the years 2000 to 2021 were included in this systematic review. The total sample size was 2500 women aged between 10-60 years. All subjects were examined by trans-perineal threedimensional ultrasound for the detection of uterine prolapse. Out of 2500 patient almost 1000 determine with uterine prolapse on ultrasound. Ultrasound findings had excellent predictive value for the diagnosis of clinical uterine prolapse. An eye - shaped vaginal canal with an antero-posterior diameter of greater than $10 \mathrm{~mm}$ in the rendered axial plane was a sign of uterine prolapse. This sign may be helpful for detecting concealed uterine prolapse in complex pelvic organ prolapse. In fact, uterine prolapse can often be more prominent on imaging than on clinical examination. This may be due to the fact that the functional anatomy of the levator hiatus seems to matter most for uterine descent.

Conclusion: From this systematic reviewed study we concluded that Ultrasound is the most appropriate form of imaging in uro-gynecology for reasons of low cost, access and performance, and due to the fact that it provides information in real time. Trans-perineal three-dimensional 2 ultrasound can evaluate the morphological changes of levator hiatus in patients with uterine prolapse, and the area of levator hiatus has high diagnostic value for uterine prolapse.
\end{abstract}

Keywords: Pelvic organ prolapse; Trans-perineal Ultrasound; Uterine Prolapse; levator ani muscles.

DOI: $10.7176 / \mathrm{JHMN} / 92-05$

Publication date:August $31^{\text {st }} 2021$

\section{Introduction}

Female pelvic organ prolapse (POP) is defined as descent of the female pelvic organs (uterus, bladder, rectum or small intestine), forming herniation of the anterior or posterior vaginal wall or vaginal apex in the vagina. ${ }^{1}$ It is generally accepted that POP is a highly prevalent disorder all over the world. ${ }^{2}$ On the one hand, more and more women are at risk. Women's lifetime risk of needing prolapse surgery is one tenth to one fifth, with over 300,000 surgeries in the USA every year. ${ }^{3}$ These symptoms include a visible or palpable vaginal bulge, or the feeling of vaginal protrusion, stress urinary incontinence, fecal incontinence, chronic straining to defecate, chronic back pain, etc. ${ }^{4}$ Among them, the first one the most particular symptom of all compartments. ${ }^{5,6}$ However, $41-50 \%$ of patients are diagnosed with certain degrees of prolapse by clinical examination and only $3-6 \%$ are diagnosed by symptoms. ${ }^{7}$

One of the pelvis organ prolapse is uterine prolapse. Uterine prolapse is defined as the falling of the womb when the muscle of the pelvis are strained to the point where they can be no longer support the position of the uterus. The uterus drop from its normal position to the pelvic cavity, descending into and in extreme condition out of the vagina. Uterine prolapse is a condition that can drastically affect quality of life causing such problems as difficulty in walking, sitting, liftingand squatting..$^{8}$

The pelvic organs are held in place by the combined actions of the levator ani muscles and connective tissue 
attachments of the organs to the pelvic sidewalls. The lateral attachment of the vagina and uterus to the pelvic sidewalls divides the pelvis into an anterior and posterior compartment. Prolapse of the pelvic organs occurs through a hiatus in the levator ani muscles. The anterior compartment contains the bladder and urethra and the medial compartment contains the uterus, fallopian tubes, ovaries, cervix, and vagina while the posterior compartment contains the rectum and anus. Normally the constant tone of the levator ani muscle holds the hiatus closed. In this situation, increases in abdominal pressure in the two compartments are equal and cancel each other out resulting in no net downward force. Birth-related levator ani muscle injury can impair the muscle's ability to maintain closure of the hiatus. This injury involves the pubo- coccygeal, but not the puborectal portion of the muscle. When this injury happens or when weak connective tissue attachment is present, the anterior or posterior vaginal wall will move downward. The vaginal walls then move downward into a position where they become exposed to a pressure differential between abdominal and atmospheric pressure. The force created by the pressure differential pulls down more on the vagina and uterus, stressing the cardinal and uterosacral ligaments. These suspending -ligaments\| are mesentery-like neurovascular structures that also contain some smooth muscle. When subjected to excessive force, over time this leads to their lengthening and further prolapse. ${ }^{9}$

Ultrasound (US) is increasingly used in evaluating women with pelvic floor dysfunction. The technique is safe, simple, inexpensive, and easily accessible to clinicians. Compared with trans- vaginal US imaging, the trans-perineal/ trans-labial approach is noninvasive and allows for assessment of pelvic floor functional anatomy on maneuvers, e.g., on Valsalva. Trans-perineal or trans-labial US (TLUS) imaging is a useful adjunct to clinical assessment of pelvic organ prolapse (POP), both in clinical audit and in research, as female POP is a common condition of poorly defined etiology that conveys significant bother. The sonographic technique is highly repeatable, both in US volume data acquisition and in offline assessment of US parameters. ${ }^{10}$

Current guidelines characterize the site of prolapse as the anterior vaginal wall, vaginal apex/uterine prolapse (apical prolapse), and posterior vaginal wall. ${ }^{11}$ Generally, ultrasound physicians adopt two methods of rating the severity or assess the possibility of POP using $2 \mathrm{D} / 3 \mathrm{D}$ ultrasound. The first one is to quantify the vertical maximal distance between the horizontal line placed through the inferior margin of the symphysis pubis and prolapsed organ on the mid- sagittal plane. The second one is to measure the antero-posterior diameter and area or other indexes of levator hiatus, sketched as the area surrounded by the pubo-visceral muscle, symphysis pubis, and inferior pubic ramus at the level of minimum hiatal dimensions on the axial plane, to estimate the degree of hiatus enlargement. ${ }^{12}$ Besides, with the development of 4D ultrasound, gaining a rendered volume of the levator ani hiatus is another method. Various studies have been conducted by diagnosing POP with the help of ultrasound and indeed improving accuracy. To our knowledge, there is no overview of all the methods.

This systematic review is a detailed and comprehensive summary of the current methods used in pelvic floor ultrasound for POP diagnosis. The aim is to evaluate how current ultrasound techniques aid POP diagnosis and to provide potential future directions for utilizing ultrasound. The uterus is more difficult to identify than bladder or rectal ampulla since it is iso-echoic, similar to vaginal muscularis. Often however, a nabothian follicle or a specular echo arising from the leading edge of the cervix, aids in identification. In fact, uterine prolapse can often be more prominent on imaging than on clinical examination. This may be due to the fact that the functional anatomy of the levator hiatus seems to matter most for uterine descent. Hence, one would expect levator co-activation to be particularly likely to result in false-negative findings of normal uterine support. In addition, trans-labial ultrasound may demonstrate the effect of an interiorized cervix in women with an enlarged, retro-verted uterus, explaining symptoms of voiding dysfunction, and supporting surgical intervention in order to improve voiding in someone with an incarcerated retro-verted fibroid uterus. ${ }^{13}$

\subsection{Method:}

Search strategy:

In this systematic review data extracted by Medline, PubMed, science direct and Obs-gyne online library that were queried for studies published between 2000 to 2021 by using specific MeSH terms.

\section{Study selection}

We extract data from 10 original articles. In this systematic article we reviewed 10 retrospective style analytical studies for the collection of data.

\subsubsection{Discussion}

Beer-Gabel et al (2002) conducted a study on -Dynamic Trans-perineal Ultrasound in the Diagnosis of Pelvic Floor Disorders.I This pilot study assessed the feasibility of dynamic trans- perineal ultrasound in a range of specific disorders affecting evacuation. A protocol of sagittal and transverse trans-perineal imaging was established defining the infra-levator viscera and soft tissues and the margins of the pubo-rectalis muscle. Dynamic measurements were possible for the extent of puborectalis shortening, the ano-rectal angle, and the movement during straining of the ano-rectal junction. Calculations were made of the depth of demonstrated 
rectoceles, the posterior urethra-vesical angle, and the movement of the urethra-vesical junction. Diagnoses were confirmed by proctography (where appropriate) and clinical examination. Transverse images of the anal sphincter were comparable with those obtained using endo-anal ultrasonography. Sagittal images permitted the measurement of puborectalis contraction and the ano-rectal angle comparable with those obtained during defecography. Cystoceles were able to be diagnosed during closure of the posterior urethra-vesical angle and abnormal urethra-vesical junction descent during straining. Rectoceles, peritoneoceles, enteroceles, and rectoanal intussusception were readily identified using dynamic trans-perineal ultrasonography. Dynamic transperineal ultrasound is a simple, noninvasive way to assess dynamically the interaction of the pelvic viscera and their relationship to the pelvic floor musculature in patients with evacuatory disorders and pelvic floor dysfunction. ${ }^{14}$

LU.R Et al (2019) conducted a study on- Application of ultrasound in diagnosis of uterine prolapse by measuring area of levator hiatus\| From September 2017 to December 2018, 80 patients diagnosed with uterine prolapse by Department of Gynecology, Xiangya Hospital of Central South University were selected as the case group, and 80 cases of normal women in the same period were selected as the control group. All subjects in both groups were examined by transperineal three-dimensional ultrasound. The anteroposterior and transverse diameters and the area of levator hiatus were measured at rest and during maximum Valsalvamaneuver respectively. The ROC curve was drawn to determine the cut-off value of area of levator hiatus in diagnosis of uterine prolapse and to evaluate its diagnostic value. At rest and during maximum

Valsalva-maneuver, the antero-posterior and transverse diameters and the area of levator hiatus in study group were larger than those in control group, the difference was statistically significant $(P<0.05)$. During maximum Valsalva maneuver, the above values in both groups were greater than those of the same group at rest state, but there was no significant difference between the two groups $(P>0.05)$. During maximum Valsalva maneuver, the best cut-off value of area of levator hiatus for the diagnosis of uterine prolapse was $22.09 \mathrm{~cm}(2)$, the area under curve was 0.893 . The sensitivity, specificity and accuracy were $90.0 \%, 95.0 \%$ and $92.5 \%$, respectively. Trans-perineal three-dimensional ultrasound can evaluate the morphological changes of levator hiatus in patients with uterine prolapse, and the area of levator hiatus has high diagnostic value for uterine prolapse. ${ }^{15}$

Dr H.P.Ditez Et al (2001) conducted a study on -Ultrasound in the quantification of female pelvic organ prolapse." Ultrasound in Obstetrics and Gynecologyll.In a prospective comparative clinical study, 145 patients referred for Uro-gynecological assessment were examined clinically by one operator and by trans-labial ultrasound by another operator. Clinical staging and International Continence Society coordinates were obtained for all 145 patients, as were ultrasound coordinates for descent of the anterior and posterior vaginal walls. Eighteen percent of the uteri of those women who had not had a hysterectomy in the past could not be seen; none of these women suffered from uterine prolapse clinically. Correlation with the prolapse assessment system recently endorsed by the International Continence Society was good $(r=0.77$ for uterine prolapse, $r=0.72$ for anterior vaginal wall and $r=0.53$ for posterior vaginal wall descent). This study demonstrates that trans-labial ultrasound can be used to quantify female pelvic organ prolapse. Correlation with the International Continence Society prolapse assessment system is good. The method may be particularly suitable for objective outcome assessment after surgical intervention. ${ }^{16}$

Tao Ying et al (2012)conductes a study on -Three-dimensional ultrasound appearance of pelvic floor in nulliparous women and pelvic organ prolapse women.\| The present study investigated the morphology and structure of pelvic floor in 50 nulliparous and 50 pelvic organ prolapse (POP) women using trans-labial threedimensional (3D) ultrasound. The levator hiatus in POP women was significantly different from that in nullipara women. In POP women, the size of

pelvic floor increased, with a circular shape, and the axis of levator hiatus departed from the normal position in $36(72 \%)$ cases. The pubo-rectalis was avulsed in $18(36 \%)$ cases and the pelvic organs arranged abnormally in $23(46 \%)$ cases. In summary, 3D ultrasound is an effective tool to detect the pelvic floor in POP women who presented with abnormalities in the morphology and structure of pelvic floor. ${ }^{17}$

R.Y.K Cheung et al (2018) conducted a study on -Pelvic organ prolapse in Caucasian and East Asian women: a comparative studyll. A total of 225 East Asian women were included between July 2012 and February 2014 from the Hong Kong clinic and 206 Caucasian women between January 2015 and July 2016 from the Sydney clinic. There was no significant difference in the overall staging of prolapse. However, in East Asian women, compared with Caucasians, apical compartment prolapse was more common $(99.6 \%$ vs $71.8 \%, P<$ $0.001)$ and posterior compartment prolapse less common $(16.9 \%$ vs $48.5 \%, P<0.001)$ on POP - Q examination. On Valsalva maneuver, the position of the uterus was lower in East Asian than in Caucasian women $(-11.3$ vs $1.35 \mathrm{~mm}, P<0.001)$, while the rectal ampulla position was lower in Caucasians than in East Asians $(-10.6 v s$ $-4.1 \mathrm{~mm}, P<0.001)$. On multiple regression analysis, Caucasian ethnicity was a significant factor for lower risk of apical compartment prolapse on clinical assessment (odds ratio $(\mathrm{OR}), 0.01 ; P<0.001)$ and on ultrasound $(\mathrm{OR}, 0.13 ; P<0.001)$, and for a higher risk of posterior compartment prolapse on clinical assessment $(\mathrm{OR}, 4.36$; 
$P<0.001)$ and of true rectocele on ultrasound (OR, 8.14; $P<0.001)$. East Asian women present more commonly with uterine prolapse while Caucasians show more often posterior compartment prolapse. Ethnicity was a significant predictor of type of prolapse on multivariate analysis. ${ }^{18}$

Ka Lai Shek Et al (2015) conducted a study on-What is abnormal uterine descent on trans-labial ultrasound \|.This was a retrospective study of patients seen for lower urinary tract symptoms and/or POP at a tertiary uro-gynecological center. All patients underwent a standardized interview, 4D TLUS and the International Continence Society Pelvic Organ Prolapse Quantification system (ICS POP-Q) assessment. Pelvic organ descent on US was measured relative to the postero-inferior margin of the symphysis pubis (SP) on maximum Valsalva. Receiver operator characteristic (ROC) statistics was used to determine the optimal cutoff. We assessed 538 data sets. Mean patient age was 53 years (range 18-88). Prolapse symptoms were reported by 263 (49\%). Clinically significant POP, i.e., ICS POP-Q stage $\geq 2$ was found in $74.5 \%$. This comprised a cystocele in 322, uterine prolapse in 63, enterocele in ten, and rectocele in 280 women. On TLUS, mean uterine position on valsalva was $14.3 \mathrm{~mm}$ above the SP. Prolapse symptoms were strongly associated with uterine descent $(20.7 \mathrm{~mm}$ vs $7.6 \mathrm{~mm}, \mathrm{P}<0.001)$. Using ROC statistics with and without excluding women with a dominant prolapse in other compartments, $+15 \mathrm{~mm}$ was found to be the optimal cutoff for predicting symptoms of prolapse, with areas under the curve of 0.68 and 0.74 , respectively. An optimal cutoff to predict prolapse symptoms due to uterine descent is a cervix descending to $15 \mathrm{~mm}$ above the symphysis pubis on maximum Valsalva. ${ }^{19}$

Gemma Blain Et al (2008) conducted a study on- Symptoms of female pelvic organ prolapse: Correlation with organ descent in women with single compartment prolapsell.A total of 1022 women were evaluated, of which 299 (29\%) reported the symptom of a vaginal lump. The area under the curve for cystocele on ultrasound and clinical examination was 0.86 and 0.89 , respectively. For rectocele, the areas were 0.81 for ultrasound and 0.89 for examination. The low number of women with an isolated uterine prolapse or enterocele limits the significance of the results for those two groups. The symptom of a vaginal lump correlates strongly with the degree of female pelvic organ prolapse as ascertained on clinical examination and ultrasound imaging. Ultrasound and clinical examination perform very well as tests for predicting symptomatic prolapse, provided that the confounding effect of other compartments is accounted for. ${ }^{20}$

Lieming Wen MD Et al (2018) conducted a study on -Detection of Concealed Uterine Prolapse in the Volume - Rendering Mode of 4 - Dimensional Trans-labial Ultrasoundll. This work was a retrospective study of 253 women who had an International Continence Society Pelvic Organ Prolapse Quantification System examination and trans-labial US examination. The antero- posterior diameter of the vaginal canal was measured in the rendered axial plane for all women. The widened vaginal canal was defined as an antero-posterior diameter above the 95th centile in women with normal uterine descent. The performance of the widened vaginal canal for prediction of clinical uterine prolapse was tested. Valid data from 233 women were analyzed. A mean antero-posterior diameter of $5.6 \mathrm{~mm}$ (range, 2.3-10.5 mm; 95th percentile, $10 \mathrm{~mm}$ ) was seen in 119 women with normal uterine descent. An eye - shaped vaginal canal with a mean antero-posterior diameter of $17.8 \mathrm{~mm}$ (range, $10.7-26.8 \mathrm{~mm}$ ) was seen in 69 women with uterine prolapse. A widened vaginal canal was defined as an antero-posterior diameter of greater than $10 \mathrm{~mm}$. Of 45 concealed uterine descents, $32 \mathrm{had}$ a widened vaginal canal (ie, antero-posterior diameter $>10 \mathrm{~mm}$ ), and 27 had clinical uterine prolapse. The $\kappa$ test showed good agreement between physical and US findings $(\kappa=0.76 ; P<.001)$. Ultrasound findings had excellent predictive value (odds ratio, 82.3) for the diagnosis of clinical uterine prolapse. An eye - shaped vaginal canal with an antero-posterior diameter of greater than $10 \mathrm{~mm}$ in the rendered axial plane was a sign of uterine prolapse. This sign may be helpful for detecting concealed uterine prolapse in complex pelvic organ prolapse. $^{21}$

Friyan Turel et al (2018) conducted a study on - Functional pelvic floor anatomy in Nepali women attending a general gynecology clinicll. one hundred and twenty-nine consecutive women attending the clinic were offered an interview, clinical examination [International Continence Society Pelvic Organ Prolapse Quantification system (ICS/POP-Q)] and 4D trans-labial ultrasound (TLUS). Most presented with general gynecological complaints. Five were excluded due to previous pelvic surgery, leaving 124.A POP-Q exam was possible in 123 women, of whom $29(24 \%)$ were diagnosed with a significant cystocele, $50(41 \%)$ significant uterine prolapse and seven (6\%) significant posterior compartment prolapse. Evaluation of 4D TLUS data sets was possible in 120 women, of whom $25(21 \%)$ had a significant cystocele, $45(38 \%)$ significant uterine prolapse and ten $(8 \%)$ significant descent of the rectal ampulla. In 13 cases, there was a rectocele with a mean depth of 14 $(10-28) \mathrm{mm}$. Of 114 women in whom uterine position could be determined, $68(60 \%)$ had a retro-verted uterus associated with significant uterine prolapse (P 0.038).POP is common in Nepali women attending a general gynaecology clinic, with a high prevalence of uterine prolapse (40\%). Uterine retroversion was seen in $60 \%$ and was associated with uterine prolapse. Patterns of POP in Nepal seem to be different from patterns observed in Western populations. $^{22}$

Dietz, H.P et al (2011) conducted a study on -Pelvic floor ultrasound in prolapse: what's in it for the 
surgeon?\| In this study he said Pelvic reconstructive surgeons have suspected for over a century that childbirthrelated trauma plays a major role in the etiology of female pelvic organ prolapse. Modern imaging has recently allowed us to define and reliably diagnose some of this trauma. As a result, imaging is becoming increasingly important, since it allows us to identify patients at high risk of recurrence, and to define underlying problems rather than just surface anatomy. Ultrasound is the most appropriate form of imaging in Uro-gynecology for reasons of cost, access and performance, and due to the fact that it provides information in real time. I will outline the main uses of this technology in pelvic reconstructive surgery and focus on areas in which the benefit to patients and clinicians is most evident. I will also try and give a perspective for the next 5 years, to consider how imaging may transform the way we deal with pelvic floor disorders. ${ }^{23}$

\subsubsection{Results}

10 studies conducted between the years 2000 to 20121 were included in this systematic review. The total sample size was 2500 women aged between 10-60 years. All subjects were examined by trans-perineal threedimensional ultrasound for the detection of uterine prolapse. Out of 2500 patient almost 1000 determine with uterine prolapse on ultrasound. The uterus is more difficult to identify than bladder or rectal ampulla since it is iso-echoic, similar to vaginal muscularis. The antero-posterior and transverse diameters and the area of levator hiatus were measured at rest and during maximum Valsalva maneuver respectively. Ultrasound findings had excellent predictive value for the diagnosis of clinical uterine prolapse. An eye - shaped vaginal canal with an antero- posterior diameter of greater than $10 \mathrm{~mm}$ in the rendered axial plane was a sign of uterine prolapse. This sign may be helpful for detecting concealed uterine prolapse in complex pelvic organ prolapse. In fact, uterine prolapse can often be more prominent on imaging than on clinical examination. This may be due to the fact that the functional anatomy of the levator hiatus seems to matter most for uterine descent. In addition, trans-labial ultrasound may demonstrate the effect of an interiorized cervix in women with an enlarged, retro-verted uterus, explaining symptoms of voiding dysfunction, and supporting surgical intervention in order to improve voiding in someone with an incarcerated retroverted fibroid uterus.

\section{Conclusion}

From this systematic reviewed study we concluded that Ultrasound is the most appropriate form of imaging in uro-gynecology for reasons of low cost, access and performance, and due to the fact that it provides information in real time. It will outline the main uses of this technology in pelvic reconstructive surgery and focus on areas in which the benefit to patients and clinicians is most evident. Dynamic trans-perineal ultrasound is a simple, noninvasive way to assess dynamically the interaction of the pelvic viscera and their relationship to the pelvic floor musculature in patients with evacuatory disorders and pelvic floor dysfunction. Trans-perineal threedimensional ultrasound can evaluate the morphological changes of levator hiatus in patients with uterine prolapse, and the area of levator hiatus has high diagnostic value for uterine prolapse.

\section{References}

1. Haylen BT, Maher CF, Barber MD, Camargo S, Dandolu V, Digesu A, et al. An International Urogynecological Association (IUGA)/ International Continence Society (ICS) joint report on the terminology for female pelvic organ prolapse (POP). Int Urogynecol J. 2016;27(4):655-84

2. Cooper J, Annappa M, Dracocardos D, Cooper W, Muller S, Mallen C. Prevalence of genital prolapse symptoms in primary care: a cross-sectional survey. Int Urogynecol J. 2015;26(4):505- 10.

3. . Wilkins MF, Wu JM. Lifetime risk of surgery for stress urinary incontinence or pelvic organ prolapse. Minerva Ginecol. 2017;69(2):171-7.

4. . Barber MD. Symptoms and outcome measures of pelvic organ prolapse. Clin Obstet Gynecol. 2005;48(3):648-61.

5. . Barber MD. Pelvic organ prolapse. BMJ. 2016;354:i3853. https:// doi.org/10.1136/bmj.i3853.

6. Manonai J, Wattanayingcharoenchai R. Relationship between pelvic floor symptoms and POP-Q measurements. Neurourol Urodyn. 2016;35(6):724-7.

7. . Barber MD, Maher C. Epidemiology and outcome assessment of pelvic organ prolapse. Int Urogynecol J. 2013;24(11)

8. Dietz, H.P. Pelvic floor ultrasound in prolapse: what's in it for the surgeon?. Int Urogynecol J 22, 12211232 (2011). https://doi.org/10.1007/s00192-011-1459-3

9. DeLancey, J. O. L. "Pelvic floor anatomy and pathology." Biomechanics of the female pelvic floor. Academic Press, 2016. 13-51.

10. Shek, K.L., Dietz, H.P. What is abnormal uterine descent on trans-labial ultrasound?. In Uro-gynecol J 26, 1783-1787 (2015). https://doi.org/10.1007/s00192-015-2792-8

11. Iglesia CB, Smithling KR. Pelvic organ prolapse. Am Fam Physician. 2017;96(3):179- 85.

12. Nyhus MO, Salvesen KA, Volloyhaug I. Association between pelvic floor muscle trauma and contraction in 
parous women from a general population - a cross sectional study. Ultrasound Obstet Gynecol. 2019;53(2):262-8. https://doi.org/10.1002/uog.19195.

13. Dietz H, Simpson J (2008) Levator trauma is associated with pelvic organ prolapse. Br J Obstet Gynaecol 115:979-984

14. Beer-Gabel, M., Teshler, M., Barzilai, N. et al. Dynamic Transperineal Ultrasound in the Diagnosis of Pelvic Floor Disorders. Dis Colon Rectum 45, 239-248 (2002).

15. Lu R, Zhang Y, Yu YP. [Application of ultrasound in diagnosis of uterine prolapse by measuring area of levator hiatus]. Zhonghua yi xue za zhi. 2019 Aug;99(29):2315-2318. DOI: 10.3760/cma.j.issn.03762491.2019.29.014.

16. Dietz, H. P., B. T. Haylen, and J. Broome. "Ultrasound in the quantification of female pelvic organ prolapse." Ultrasound in Obstetrics and Gynecology: The Official Journal of the International Society of Ultrasound in Obstetrics and Gynecology 18.5 (2001): $511-514$.

17. Ying, Tao, et al. "Three-dimensional ultrasound appearance of pelvic floor in nulliparous women and pelvic organ prolapse women." International journal of medical sciences 9.10(2012): 894.

18. R. Y. K. Cheung S. S. C. Chan K. L. Shek T. K. H. Chung H. P. Dietz,Pelvic organ prolapse in Caucasian and East Asian women: a comparative study,23 September 2018 https://doi.org/10.1002/uog.20124

19. Shek, K.L., Dietz, H.P. What is abnormal uterine descent on trans-labial ultrasound?. Int Urogynecol J 26, 1783-1787 (2015). https://doi.org/10.1007/s00192-015-2792-8

20. Blain, Gemma, and Hans Peter Dietz. "Symptoms of female pelvic organ prolapse: correlation with organ descent in women with single compartment prolapse." Australian and New Zealand Journal of Obstetrics and Gynecology 48.3 (2008): 317-321.

21. Wen, Lieming, et al. "Detection of Concealed Uterine Prolapse in the Volume - Rendering Mode of 4 Dimensional Trans-labial Ultrasound: A Retrospective Observational Study." Journal of Ultrasound in Medicine 38.7 (2019): 1705-1711.

22. Turel, F., Caagbay, D. \& Dietz, H.P. Functional pelvic floor anatomy in Nepali women attending a general gynaecology clinic. Int Urogynecol J 29, 1435-1440 (2018). https://doi.org/10.1007/s00192-017-3534-x

23. Dietz, H.P. Pelvic floor ultrasound in prolapse: what's in it for the surgeon?. Int Urogynecol J 22, 12211232 (2011). https://doi.org/10.1007/s00192-011-1459-3 